\title{
Correlation between the Angle of the Guide Plate and Crushing Performance in Vertical Shaft Crushers
}

\author{
Feifei Feng $(\mathbb{D}$, Jinfa Shi, Jie Yang, and Junxu Ma \\ North China University of Water Resources and Electric Power, Zhengzhou 450045, China \\ Correspondence should be addressed to Feifei Feng; 1573865218@qq.com
}

Received 1 December 2021; Revised 16 January 2022; Accepted 1 February 2022; Published 27 February 2022

Academic Editor: Letícia Fleck Fadel Miguel

Copyright (c) 2022 Feifei Feng et al. This is an open access article distributed under the Creative Commons Attribution License, which permits unrestricted use, distribution, and reproduction in any medium, provided the original work is properly cited.

\begin{abstract}
A geometric model of rotors is established on the basis of the structure of vertical shaft impact crusher. The dynamic simulation of the rotor is carried out by using the discrete element software EDEM. At the same time, the correlation between the angle of the guide plate and the crushing performance of the crusher is studied systematically. The optimal angle of the guide plate is obtained by theoretical analysis of the particle motion. The simulation method is used to analyze the velocity of particles, so the influence law of the angle of the guide plate on the acceleration effect of particles is obtained. The results of this paper show that the peak value of the maximum velocity of particles is the highest when the angle of the guide plate is $35^{\circ}$, which is consistent with the theoretical results. At this time, the average velocity of particle population is the largest and the proportion of particles with high velocity is the highest. It means that the particles obtain the largest crushing kinetic energy and the best crushing performance.
\end{abstract}

\section{Introduction}

In recent years, the demand for sands has increased in the construction industry, road transportation, and other infrastructure construction. The vertical shaft impact crusher is one of the main equipment for sand crushing, and it has the advantages of simple structure, convenient maintenance, and stable operation [1,2]. Figure 1 shows a schematic diagram of the structure of the vertical shaft impact crusher. It is mainly composed of spindle assembly, motor, rotor, crushing cavity, feed hopper, and control system [3]. Under the action of centrifugal force, the particles leave the rotor at a certain speed and collide with the crushing cavity to achieve the purpose of crushing. The greater the velocity of particles colliding with the crushing cavity, the greater the energy of particles will be and the better the crushing effect will be. If the acceleration of particles is not sufficient, it will cause problems such as low sand formation rate. However, the particles are mainly accelerated, collided, and ground on the guide plate.

In 1971, Cundall put forward the discrete element method, which is widely used in geotechnical engineering, aerospace, chemical industry, and other fields [4]. Zhang and
$\mathrm{Vu}-\mathrm{Quoc}$ [5] simulated the velocity distribution of particle flow. Segura-Salazar et al. [6] established the mathematical model of the vertical impact crusher based on the Whiten model. After that, the discrete element method was used to simulate the flow of particles in the rotor, and the influence of feed rate on the performance of the crusher was studied. Djordjevic et al. [7] used the discrete element method to simulate the vertical impact crusher in detail. The effects of mechanical design and operating conditions on the collision velocity, energy distribution, and particle crushing behavior in the milling chamber were studied. Duan et al. [8] used EDEM to conduct dynamic simulation research on the $3 \mathrm{D}$ model of the new rotor. The results showed that the installation angle of the guide plate has no significant effect on the second acceleration. Cleary and Sinnott and $[9,10]$ used DEM to simulate particle flow in the crusher and estimated the stress applied by particles flowing through the crusher. The study by da Cunha et al. [11] showed that DEM can be used to predict the breakage energy and damage frequency of VSI.

The research results of these scholars show that the simulation results of the vertical shaft impact crusher using the discrete element method are feasible. Therefore, to 


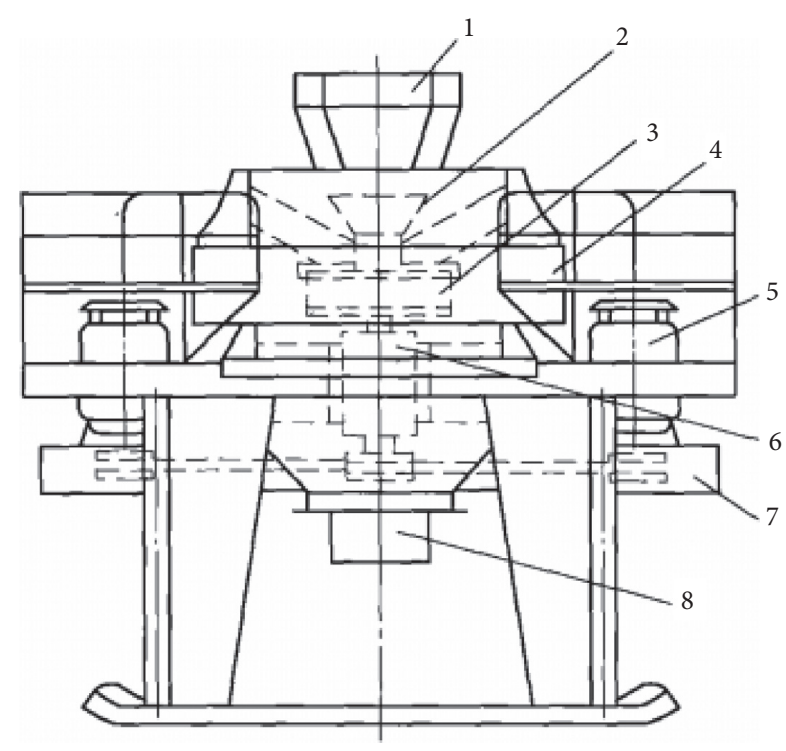

FIGURE 1: The vertical shaft impact crusher. (1) Feeder; (2) splitter; (3) rotor; (4) crushing cavity; (5) motor; (6) spindle assembly; (7) rack; (8) hopper.

improve the crushing performance of the vertical shaft impact crusher, this paper studies the particle crushing performance on the basis of the discrete element method. To explore the influence of the angle of the guide plate on the crushing performance of the crusher, the maximum velocity, average velocity, and velocity distribution of the particles are analyzed. It provides a certain reference for rotor structure design and engineering optimization.

\section{The Crushing Mechanism of the Vertical Shaft Impact Crusher}

2.1. Rotor Model. The rotor is an important part of the vertical shaft impact crusher. There are two categories: openloop rotor and closed-loop rotor. Figure 2 shows a threedimensional model of the rotor, which is mainly composed of feeding ring, split cone, guide plate, and upper and lower wear-resistant plates [12]. Among them, the split cone is located in the center of the rotor and is usually made of hard alloy, high-chromium cast iron, and other materials. During the operation of the crusher, the particles inside the rotor are evenly distributed to both sides of the guide plate by the split cone. After accelerating on the guide plate, the particles leave the rotor and collide with the crushing cavity to achieve crushing.

2.2. Motion Analysis of Particles. If the particle leaves the rotor, its absolute velocity will be very high. Therefore, the particle is more likely to be broken when it collides with the crushing cavity. The guide plate can be installed in three ways: forward guide plate, radial guide plate, and backward guide plate. From the point of view of particle velocity, the velocity of the particles leaving the rotor is the highest when

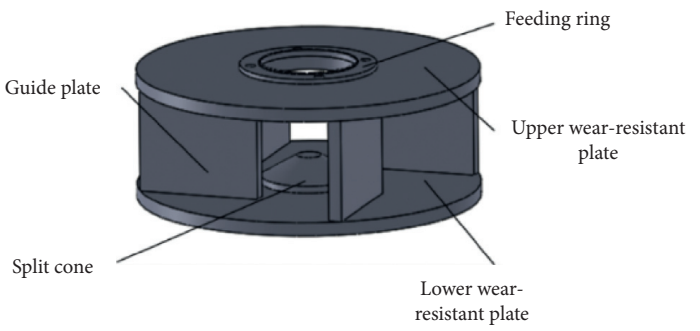

FIgure 2: Three-dimensional model of the rotor.

using the installation mode of the forward guide plate, followed by the radial guide plate. From the point of view of wear, the wear of the forward guide plate is the most serious, while the wear of the backward guide plate is lighter [13]. Considering comprehensively, the installation mode of the forward guide plate is adopted in this paper.

Figure 3 shows a schematic diagram of the motion of particles on the guide plate. In Figure 3, $r_{0}$ is the radius of the split cone, $r_{1}$ is the radius of the rotor, and $\theta$ is the angle of the guide plate, that is, the angle between the guide plate and the radial direction $(\theta \geq 0)$.

When the rotor moves in a circle, the particle produces the entrainment velocity $\left(\overrightarrow{v_{e}}\right)$ at point $\mathrm{A}$. It is also the linear velocity of the rotor at point $A$, which can be calculated by the following formula:

$$
v_{e}=\omega r_{1},
$$

where $\omega$ is the angular velocity of the rotor.

The relative velocity $\left(\overrightarrow{v_{r}}\right)$ is the velocity of the particle relative to the guide plate, and its direction is along the guide plate. As can be seen from Figure 3, the angle between the relative velocity direction and the radial direction of the particle at point $\mathrm{A}$ is $\theta$, which is equal to the angle of the guide plate. The calculation formula of relative velocity is as follows:

$$
v_{r}=\left[\left(1+f^{2}\right)^{1 / 2}-f\right] \omega r_{1}(\cos \theta+f \sin \theta),
$$

where $f$ is the friction coefficient of the guide plate.

Let

$$
\begin{aligned}
& a=\left(1+f^{2}\right)^{1 / 2}-f, \\
& b=\cos \theta+f \sin \theta .
\end{aligned}
$$

When the material of the guide plate is determined, its friction coefficient is a constant. It can be known that $a$ is a constant and $b$ is only related to the angle of the guide plate.

The absolute velocity of the particle leaving the rotor is $\vec{v}$. It can be obtained by the following formula:

$$
\vec{v}=\overrightarrow{v_{e}}+\overrightarrow{v_{r}}
$$

According to the geometric relationship between absolute velocity, relative velocity, and entrainment velocity of the particle, it can be known that 


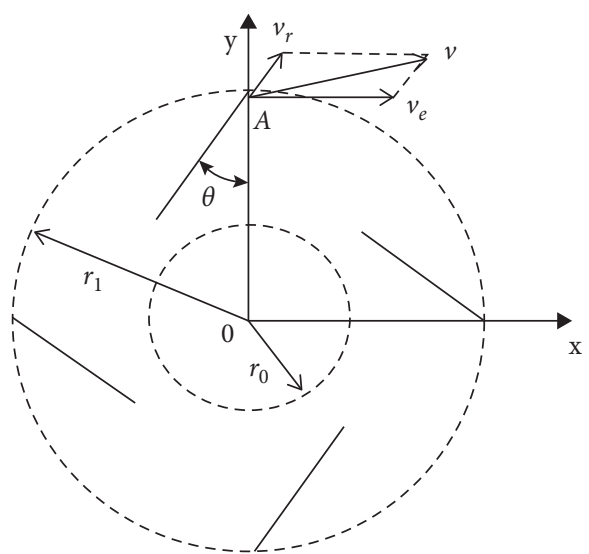

FIGURE 3: Schematic diagram of the motion of particles on the guide plate.

$$
v^{2}=v_{e}^{2}+v_{r}^{2}-2 v_{e} v_{r} \cos \left(\frac{\pi}{2}+\theta\right)
$$

The absolute velocity of the particle leaving the rotor can be obtained from formulas $(1) \sim(6)$ :

$$
v=\omega r_{1} \sqrt{1+a^{2} b^{2}-2 a b \cos \left(\frac{\pi}{2}+\theta\right)} .
$$

After the particle is accelerated by the guide plate, it leaves the rotor at a certain angle. By adjusting the installation angle of the lining plate of the crushing cavity, the particle is approximately vertically impacted on the lining plate. At this point, the higher the velocity of particle leaving the rotor, the better the crushing effect.

It can be seen from formula (7) that the absolute velocity of particles leaving the rotor is mainly related to the friction coefficient of the guide plate, the rotor speed, the rotor radius, and the angle of the guide plate. There are many factors to be considered in the material selection of the guide plate, but it is easy to determine the material of the guide plate in the design and manufacture. When the angle of the guide plate is constant, the higher the rotation speed of the rotor and the higher the velocity of the particles. However, with the increase in rotational speed, it is necessary to put forward higher requirements for the manufacturing process of the equipment. In addition, the increase in rotational speed will lead to the vibration of the whole machine and accelerate the wear of the guide plate. If the rotational speed is constant, increasing the radius of the rotor can also increase the velocity of the particles. However, with the increase in the structure and size of equipment, the manufacturing cost of equipment increases.

\section{Simulation Principle and Parameter Setting}

3.1. Defining Model Parameters. The three-dimensional model of the rotor was created by using the three-dimensional software SolidWorks and was imported into EDEM 2018 version. Among them, the radius of the split cone is $r_{0}=70 \mathrm{~mm}$, the radius of the guide plate is $r_{1}=250 \mathrm{~mm}$, the number of the guide plate is $m=4$, and the height of the guide plate is $H=120 \mathrm{~mm}$. The simulation area of the rotor is shown in Figure 4, where the area within the red frame is the calculation area of EDEM.

We use the Particle Factory tool to add a Particle Factory and set the Particle Factory as a cylinder with a diameter of $135 \mathrm{~mm}$ and a height of $120 \mathrm{~mm}$. The material type of the Particle Factory was set as virtual and coaxial with the rotor.

3.2. Defining Material Properties. In EDEM, the material of the particles was set to limestone and the material of the rotor was set to steel. We can refer relevant information to know the material properties of the particles and the rotor as shown in Table 1 and the contact parameters between the particles and the rotor as shown in Table 2 [13].

3.3. Particle Contact Model. The basic idea of the discrete element method is to separate the discontinuous body into a set of rigid body elements, so that the whole motion form of the discontinuous body can be obtained by solving the motion equation of each rigid body element [14].

The contact model is the basis of the discrete element method, which is used to determine the force and moment of the particles. There are mainly two kinds of particle models: soft sphere model and hard sphere model. Because the deformation of particles is not considered in this paper, the hard sphere model is chosen.

Figure 5 shows a particle contact model. The contact force between two particles includes normal force and tangential force. The normal force is composed of damping force and elastic force, which is equivalent to an elasticdamping system. The calculation of tangential force includes elastic force, damping force, and friction force $[15,16]$.

The normal force between particles is $F_{n}$, which can be calculated by the following formula:

$$
F_{n}=\frac{4}{3} E_{1,2}^{*}\left(R_{1,2}^{*}\right)^{1 / 2} \alpha^{3 / 2},
$$

where $E_{1,2}^{*}$ is the equivalent elastic modulus of the two particles; $R_{1,2}^{*}$ is the equivalent particle radius of the two particles; and $\alpha$ is the normal overlap of the two particles.

The normal damping force between particles is $F_{n}^{d}$, which can be calculated by the following formula:

$$
F_{n}^{d}=-2 \sqrt{\frac{5}{6}} \beta \sqrt{K_{n} m_{1,2}^{*}} v_{n}^{\text {rel }},
$$

where $m_{1,2}^{*}$ is the equivalent mass of two particles and $v_{n}^{\text {rel }}$ is the normal component of the relative velocity.

In formula (9), the coefficient $\beta$ is the parameter related to the restitution coefficient and the coefficient $K_{n}$ is the normal rigidity, which can be obtained from the following formula, respectively:

$$
\begin{gathered}
\beta=\frac{\ln e}{\sqrt{\ln ^{2} e+\pi^{2}}} \\
K_{n}=2 E_{1,2}^{*} \sqrt{R_{1,2}^{*} \alpha},
\end{gathered}
$$

where $e$ is the restitution coefficient. 


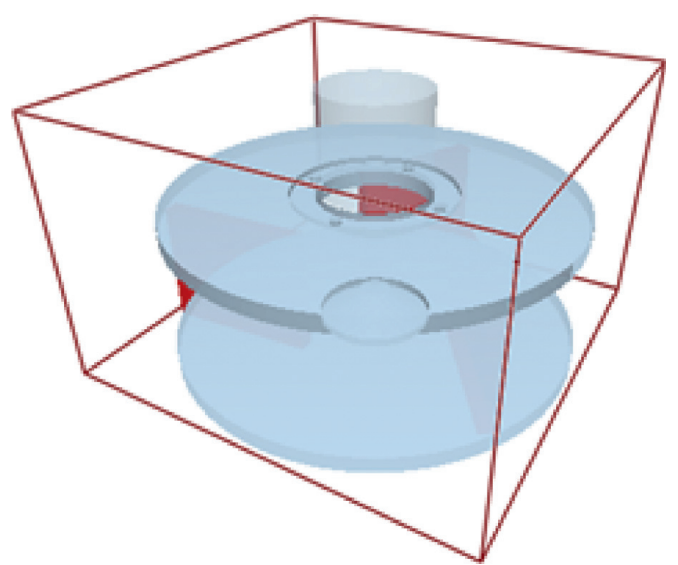

Figure 4: Computing domain of EDEM.

TABLE 1: Material property table.

\begin{tabular}{lccc}
\hline Materials & Poisson's ratio & $\begin{array}{c}\text { Density }(\mathrm{kg} / \\
\left.\mathrm{m}^{3}\right)\end{array}$ & Shear modulus (Pa) \\
\hline Limestone & 0.25 & 2640 & $2.09 \times 10^{8}$ \\
Steel & 0.30 & 7800 & $7 \times 10^{8}$ \\
\hline
\end{tabular}

The tangential force between particles is $F_{t}$, which can be calculated by the following formula:

$$
F_{t}=-K_{t} \delta,
$$

where $\delta$ is the tangential overlap of the two particles.

In formula (12), the coefficient $K_{t}$ is the tangential rigidity, which can be obtained from the following formula:

$$
K_{t}=8 G_{1,2}^{*} \sqrt{R_{1,2}^{*} \alpha}
$$

where $G_{1,2}^{*}$ is the equivalent shear modulus.

Therefore, the tangential damping force $F_{t}^{d}$ between the two particles can be obtained as follows:

$$
F_{t}^{d}=-2 \sqrt{\frac{5}{6}} \beta \sqrt{K_{t} m_{1,2}^{*}} v_{t}^{\text {rel }}
$$

where $v_{t}^{\text {rel }}$ is the tangential component of the relative velocity.

Therefore, the Hertz-Mindlin (no slip) contact model $[17,18]$ was adopted for the collisions among particles and between particles and the rotor in EDEM.

3.4. Defining Simulation Parameters. In reality, each particle is different in shape and size. Therefore, the shape of the particles was simplified and set as a sphere with a diameter of $10 \mathrm{~mm}$ in the simulation process. However, particle breakage was not taken into account in order to improve the efficiency of simulation. The initial velocity of particles leaving the particle factory was $1 \mathrm{~m} / \mathrm{s}$. The particle factory generated a total of 2,000 particles at a rate of 500 particles per second. The rotor speed was set to $n=2000 \mathrm{r} / \mathrm{min}$. The simulation time was set to $5 \mathrm{~s}$.

\section{Test and Analysis}

4.1. Calculation of the Theoretical Velocity of Particles. According to the previous analysis of the acceleration principle of particles, the size parameters of the rotor and the friction coefficient between the particle and the rotor are brought into formulas (1) (7). It is known that when the angle of the guide plate is $\theta=34.85^{\circ}$, the velocity of the particle leaving the rotor is the maximum, which is $v=83.80 \mathrm{~m} / \mathrm{s}$

4.2. Motion Velocity Analysis of Particles. The theoretical analysis shows that the particle velocity is the maximum when the angle of the guide plate is $\theta=34.85^{\circ}$. Therefore, the angles of the guide plate were selected as $25^{\circ}, 30^{\circ}, 35^{\circ}, 40^{\circ}$, and $45^{\circ}$ to carry out the simulation test.

As shown in Figure 6, a collection area was designed around the rotor. At the same time, the cell rotated synchronously with the rotor.

4.2.1. Maximum Velocity Analysis of Individual Particles. The maximum velocity of particles in each time step was extracted by using the postprocessing function of EDEM. It should be pointed out that the velocity is the maximum velocity of a certain particle among all particles in the rotor [19]. However, the particle with the maximum velocity at each moment may not be the same, and the maximum velocity of the individual particles changes with the rotation of the rotor. Figure 7 shows the variation law of the maximum individual particle velocity over time.

In order to analyze the acceleration ability of the rotor to the individual particles, the maximum velocity of all particles at each time step is extracted in the velocity collection area and the maximum value point is obtained, which is called the peak. At the same time, the average value of the maximum velocity of all particles at each time step is obtained. It is used to analyze the acceleration ability of the rotor to the particles as a whole in the whole process.

Table 3 shows the peak and average of the maximum velocity of the particles during the whole simulation process.

It can be seen from Table 3 that when the angle of the guide plate is $35^{\circ}$, the peak value of the maximum velocity of the individual particle is $104.43 \mathrm{~m} / \mathrm{s}$ and the average value of the maximum velocity of the particles is $86.83 \mathrm{~m} / \mathrm{s}$. It is obvious that the particle performs best under the condition of different guide plate angles.

According to the previous theoretical calculation, when the angle of the guide plate is $\theta=35^{\circ}$, the maximum velocity of the particle is $83.79 \mathrm{~m} / \mathrm{s}$. The angle of the guide plate is basically consistent with the theoretical calculation results. It can be seen that the maximum velocity of individual particles in the simulation test is larger than the theoretical maximum velocity of individual particles, which is mainly caused by two reasons.

First, the collisions among particles and between particles and the split cone are not considered in the theoretical calculation. Second, in the simulation process of EDEM, considering the material properties of particles and the rotor, the 
TABle 2: Contact parameter table.

Contact materials

Limestone and limestone

Limestone and steel

\section{Restitution coefficient}

0.207

0.557
Static friction coefficient

0.77

0.61

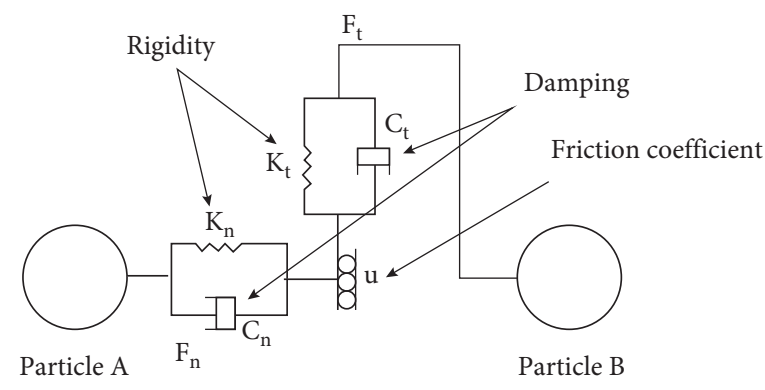

Figure 5: Particle contact model.

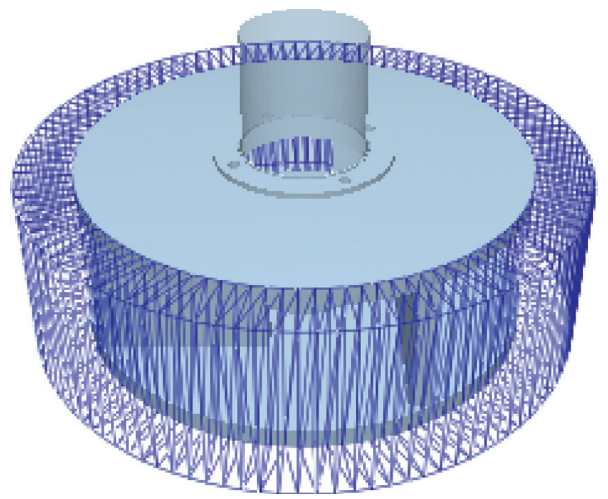

Figure 6: Velocity collection area.

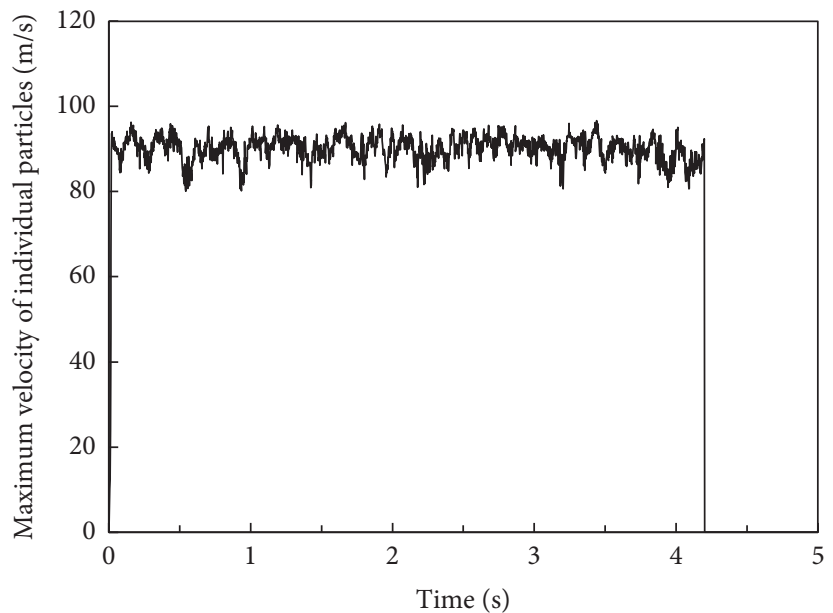

FIGURE 7: Variation law of the maximum individual particle velocity over time. 
TABLE 3: Maximum velocity of individual particles.

\begin{tabular}{lcc}
\hline Angle of the guide plate $\left({ }^{\circ}\right)$ & Peak of maximum velocity $(\mathrm{m} / \mathrm{s})$ & Average of maximum velocity $(\mathrm{m} / \mathrm{s})$ \\
\hline 25 & 96.61 & 84.72 \\
30 & 98.37 & 86.28 \\
35 & 102.43 & 88.83 \\
40 & 97.81 & 86.97 \\
45 & 95.43 & 85.16 \\
\hline
\end{tabular}

TABLe 4: Average velocity of the particle population.

\begin{tabular}{lcc}
\hline Angle of the guide plate $\left(^{\circ}\right)$ & Average velocity $(\mathrm{m} / \mathrm{s})$ & Standard deviation $(\mathrm{m} / \mathrm{s})$ \\
\hline 25 & 75.24 & 7.58 \\
30 & 79.58 & 8.62 \\
35 & 80.87 & 7.11 \\
40 & 78.51 & 6.74 \\
45 & 77.15 & 7.32 \\
\hline
\end{tabular}

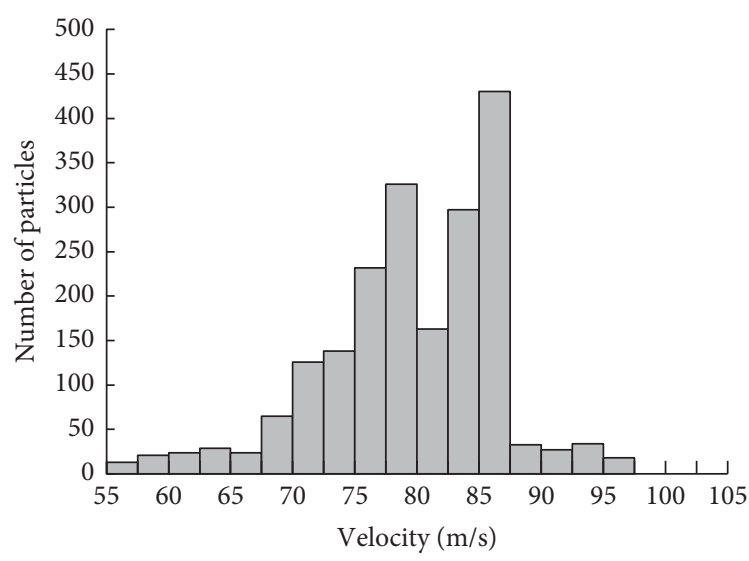

(a)

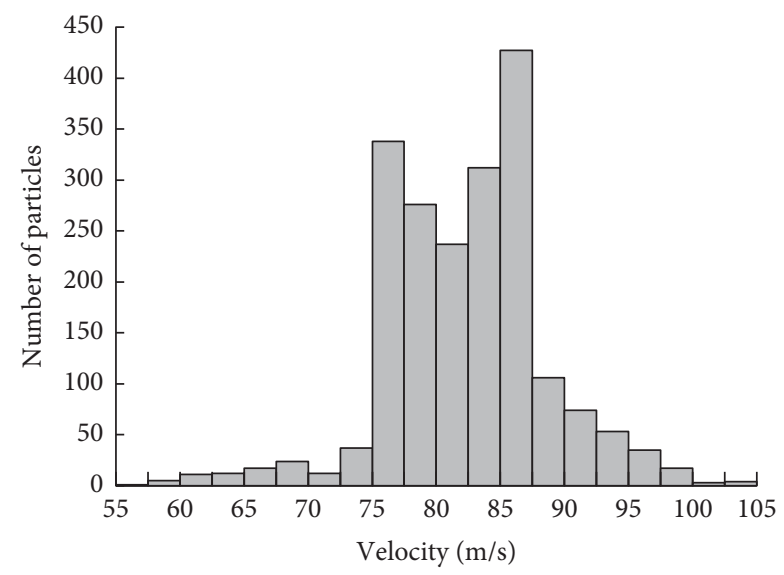

(c)

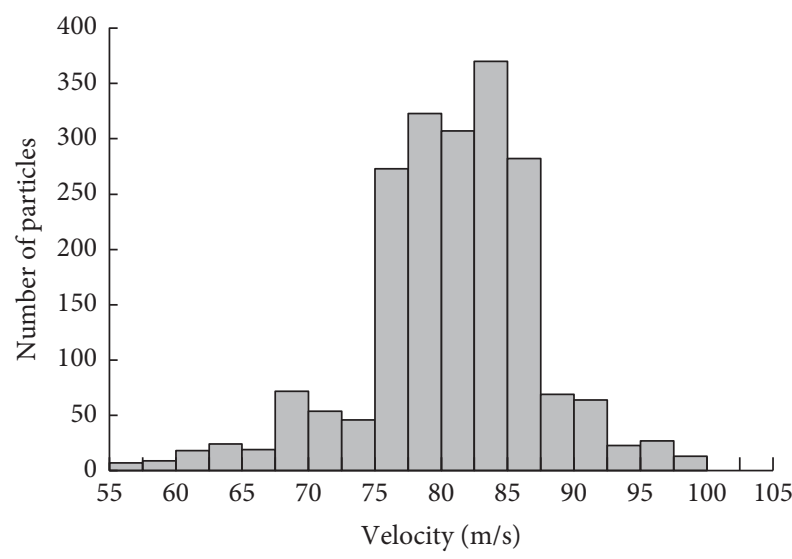

(b)

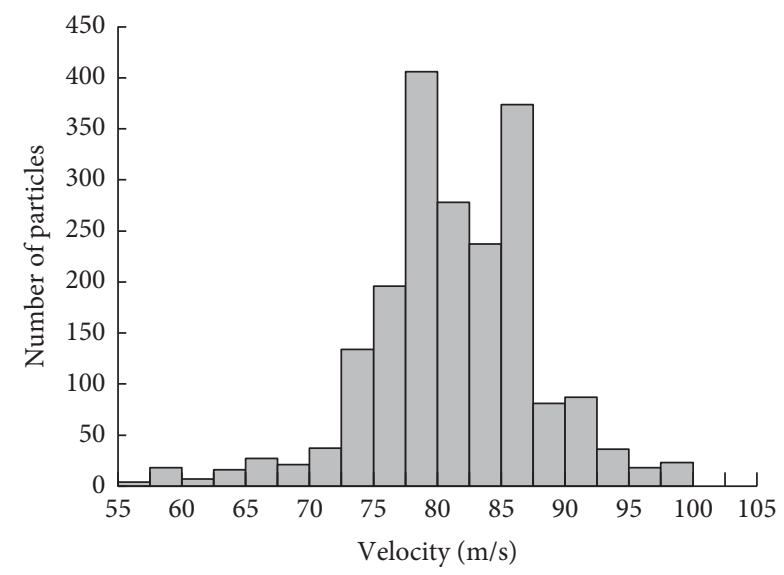

(d)

FIgURE 8: Continued. 


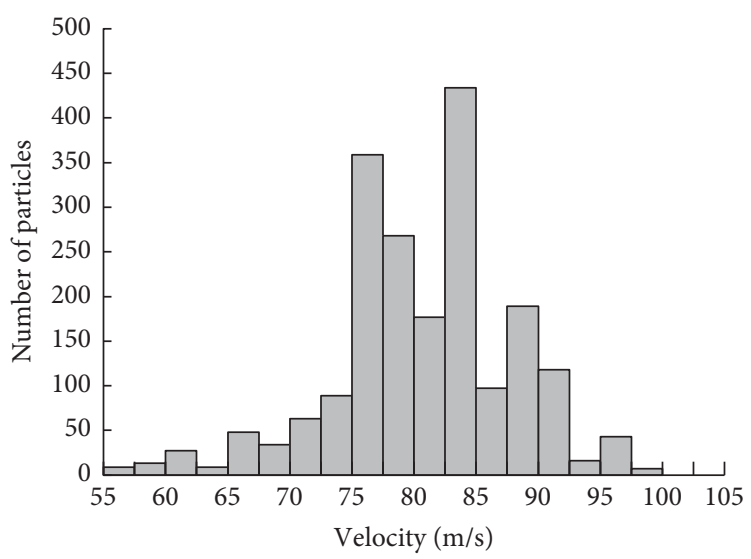

(e)

Figure 8: Histogram of population velocity distribution of particles. The angle of the guide plate: (a) $25^{\circ}$; (b) $30^{\circ}$; (c) $35^{\circ}$; (d) $40^{\circ}$; (e) $45^{\circ}$.

TABLE 5: Velocity distribution of the particle population.

\begin{tabular}{lc}
\hline Angle of the guide plate $\left({ }^{\circ}\right)$ & Ratio of particles with velocity between $80 \mathrm{~m} / \mathrm{s}$ and $105 \mathrm{~m} / \mathrm{s}$ \\
\hline 25 & 0.501 \\
30 & 0.578 \\
35 & 0.634 \\
40 & 0.567 \\
45 & 0.541 \\
\hline
\end{tabular}

momentum transformation at the moment of collision between particles and the rotor is calculated in real time. In the theoretical calculation, only the acceleration of particles with the guide plate is considered, so the theoretical results of particle velocity are inconsistent with the simulation results.

\subsubsection{Average Velocity Analysis of Particle Population.}

The maximum velocity of the individual particles can only reflect the acceleration ability of the rotor to the particle. For the performance of the rotor, the velocity of all particles should be considered. The higher the velocity of the particle population, the greater the total kinetic energy it has and the better the particle crushing effect. Therefore, the average velocity of the particle population can be used to study the acceleration performance of the rotor to some extent. The velocity of particle population in the acceleration area at each moment was extracted, and the data were analyzed. The analysis results are shown in Table 4.

As can be seen from Table 4, when the angle of the guide plate is $35^{\circ}$, the average velocity of the particle population is the maximum. When the angle of the guide plate is $40^{\circ}$, the standard deviation of the particle population velocity is the smallest, which indicates that the velocity uniformity of the particle population in the acceleration area is the best at this time, followed by the angle of the guide plate at $35^{\circ}$.

Therefore, the angle of the guide plate should be selected as $35^{\circ}$ after comprehensive consideration.

4.2.3. Velocity Distribution of Particle Population. To further analyze the influence of the angle of the guide plate on the acceleration performance of the rotor, the velocity distribution of particles leaving the rotor was studied.

The postprocessing function of EDEM is used to extract the velocity of particles leaving the rotor. The average velocity of the particle population and the standard deviation of the velocity distribution can be known from Table 4 . After statistical analysis of the velocity data of the particle population, the results are shown in Figure 8. The number of particles in different particle velocity ranges can be clearly seen from Figure 8.

The number of particles in each particle velocity range is counted when the particles leave the rotor. In the case of different guide plate angles, the ratio of the number of particles to the total number of particles between the particle velocity of $80 \mathrm{~m} / \mathrm{s}$ and $105 \mathrm{~m} / \mathrm{s}$ can be obtained. The statistical results are shown in Table 5 .

The analysis of Figure 8 and Table 5 shows that when the angle of the guide plate is $35^{\circ}$, the velocity distribution of particles is relatively concentrated and the number of highspeed particles is relatively large, accounting for $63.4 \%$ of the total particles. The more the number of high-speed particles is, the greater the impact kinetic energy of the particles will be and the better the crushing effect will be.

\section{Conclusions}

Based on the discrete element method, the rotor of the vertical shaft impact crusher was simulated. The influence of different guide plate angles on rotor acceleration performance was studied by extracting the velocity data of particles. The main conclusions are as follows: 
(1) When the angle of the guide plate was $35^{\circ}$, the maximum velocity peak value of the particles in the whole process was relatively high, which was consistent with the theoretical calculation results. At the same time, it proved the rationality of the velocity deduction. Moreover, the average maximum velocity at this time was also higher than that of the guide plate at other angles.

(2) When the angle of the guide plate was $35^{\circ}$, the average velocity of the particle population was the largest and the standard deviation of the particle population velocity was relatively small, which indicated that the particle velocity distribution of particles was more uniform at this time. When the angle of the guide plate was $40^{\circ}$, the standard deviation of particle population velocity was the smallest.

(3) Through the study of the particle velocity distribution of particles, it was known that when the angle of the guide plate was $35^{\circ}$, the velocity distribution of particles is relatively concentrated. At the same time, the particles between $80 \mathrm{~m} / \mathrm{s}$ and $105 \mathrm{~m} / \mathrm{s}$ account for a large proportion, indicating that the acceleration effect of particles was relatively good. It can be seen that the large proportion of particles in the highspeed part provided some data support for rotor structure optimization and provided design reference for engineering optimization.

\section{Data Availability}

No data were used to support the findings of this study.

\section{Conflicts of Interest}

The authors declare that there are no conflicts of interest regarding the publication of this paper.

\section{Acknowledgments}

This project was supported by the National Natural Science Foundation of China: Research on Enterprise Resource Location Optimization Based on the Internet of things (71371172) and Henan 2018 Science and Technology Project: Key Technologies and Development of Big Data Based Intelligent Control System and Production Management Platform for Mixing Plant (182102210060).

\section{References}

[1] R. Cepuritis, S. Jacobsen, and T. Onnela, "Sand production with VSI crushing and air classification: optimising fines grading for concrete production with micro-proportioning," Minerals Engineering, vol. 78, pp. 1-14, 2015.

[2] M. Bengtsson, "Per Svedensten, and C. Magnus Evertsson, "Improving yield and shape in a crushing plant," Minerals Engineering, vol. 22, no. 7, pp. 618-624, 2009.

[3] H. Fang, J. Yang, Y. Song, W. Huang, and J. Chen, "Simulation and experimental study on the stone powder separator of a vertical shaft impact crusher," Advanced Powder Technology, vol. 31, no. 3, pp. 1013-1022, 2020.

[4] W. Cui, J. Wei, and G. ke Zhang, "Research on collapse characteristics of binary particle column based on discrete element simulation," Rock and Soil Mechanics, vol. 42, no. 1, pp. 280-290, 2021.

[5] X. Zhang and L. Vu-Quoc, "Simulation of chute flow of soybeans using an improved tangential force-displacement model," Mechanics of Materials, vol. 32, no. 2, pp. 115-129, 2000.

[6] J. Segura-Salazar, G. P. Barrios, V. Rodriguez, and L. M. Tavares, "Mathematical modeling of a vertical shaft impact crusher using the Whiten model," Minerals Engineering, vol. 111, pp. 222-228, 2017.

[7] N. Djordjevic, F. N. Shi, and R. D. Morrison, "Applying discrete element modelling to vertical and horizontal shaft impact crushers," Minerals Engineering, vol. 16, no. 10, pp. 983-991, 2003.

[8] D. R. Duan, F. Zhao, S. Wang, and X. X. Chen, "Research of the installation angle of new rotor impact plate based on EDEM," Applied Mechanics and Materials, vol. 80-81, pp. 1133-1137, 2011.

[9] P. W. Cleary and M. D. Sinnott, "Simulation of particle flows and breakage in crushers using DEM: Part 1 - compression crushers,” Minerals Engineering, vol. 74, pp. 178-197, 2015.

[10] M. D. Sinnott and P. W. Cleary, "Simulation of particle flows and breakage in crushers using DEM: Part 2 - impact crushers," Minerals Engineering, vol. 74, pp. 163-177, 2015.

[11] E. R. da Cunha, R. M. de Carvalho, L. M. Tavares, and L. MarceloTavares, "Simulation of solids flow and energy transfer in a vertical shaft impact crusher using DEM," Minerals Engineering, vol. 43-44, no. 44, pp. 85-90, 2013.

[12] S. Nikolov, "Modelling and simulation of particle breakage in impact crushers," International Journal of Mineral Processing, vol. 74, pp. s219-s225, 2004.

[13] T. A. O. Yin, "FANG Huaiying. YANG Jianhong, "Simulation and analysis on wear of accelerating board of vertical-shaft impact crusher," Mining \& Processing Equipment, vol. 45, pp. 37-42, 2017.

[14] M. Manuel Moncada and G. Cristian, "Rodríguez, giuseppe ruta, "dynamic modeling of a vibrating screen considering the ore inertia and force of the ore over the screen calculated with discrete element method," Shock and Vibration, vol. 2018, Article ID 1714738, 13 pages, 2018.

[15] C. Santos, V. Urdaneta, and X. García, "Compression and shear-wave velocities in discrete particle simulations of quartz granular packings: improved Hertz-Mindlin contact model," Geophysics, vol. 76, no. 5, 2011.

[16] E. Olsson and D. Jelagin, "A contact model for the normal force between viscoelastic particles in discrete element simulations," Powder Technology, vol. 342, pp. 985-991, 2018.

[17] S. Carlos, U. Vanessa, and G. Xavier, "Compression and shear-wave velocities in discrete particle simulations of quartz granular packings: improved Hertz-Mindlin contact model," Geophysics, vol. 76, no. 5, pp. E165-E174, 2011.

[18] S. J. Burns, P. T. Piiroinen, and K. J. Hanley, "Critical time step for DEM simulations of dynamic systems using a Hertzian contact model," International Journal for Numerical Methods in Engineering, vol. 119, no. 5, pp. 432-451, 2019.

[19] N. Stoimenov and J. Ruzic, "Analysis of the particle motion during mechanical alloying using EDEM software," IFACPapersOnLine, vol. 52, no. 25, pp. 462-466, 2019. 